\title{
Equivalent Circuit Modelling of Travelling Wave Accelerating Structures and Its Applications
}

\author{
Nasrin Nasr Esfahani ${ }^{1}$, Patrick Kramer ${ }^{* 2}$, and Christine Vollinger ${ }^{3}$ \\ CERN, European Organization for Nuclear Research, Geneva, Switzerland \\ ${ }^{1}$ Nasrin.Nasresfahani@cern.ch, ${ }^{2}$ Patrick.Kramer@cern.ch, ${ }^{3}$ Christine.Vollinger@cern.ch \\ ${ }^{* 2}$ Institute of High Frequency Technology (IHF), RWTH Aachen University, Germany
}

\begin{abstract}
This paper presents an equivalent circuit model for the parallel bar $200 \mathrm{MHz}$ travelling wave structure of the CERN Super Proton Synchrotron. This equivalent circuit will be used for studying the performance of the higher order mode couplers at $628 \mathrm{MHz}$. Currently, this mode presents a serious limitation for future beam intensities required for high luminosity LHC operation.
\end{abstract}

\section{INTRODUCTION}

In recent years, almost the entire LHC (Large Hadron Collider) injector chain at $\mathrm{CERN}^{1}$, including the super proton synchrotron (SPS) with its $200 \mathrm{MHz}$ travelling wave cavities has been subject to various beam dynamic and RF studies (see, e.g. [1]) conducted in the framework of the LHC Injectors Upgrade (LIU) project. From these studies, it can be seen that the $628 \mathrm{MHz}$ higher order mode (HOM) within the $200 \mathrm{MHz}$ accelerating cavity will be a source of instabilities at higher beam intensities. Therefore, further damping of this unwanted mode through modification of the existing $628 \mathrm{MHz}$ couplers or introducing new HOM couplers is currently an important task in the upgrade program.

Today, there are two four-section and two five-section cavities $^{2}$ installed in the SPS ring. Each section consists of 11 single-cells and is equipped with four $628 \mathrm{MHz} \mathrm{HOM}$ couplers (see Figs. 1 and 5 (c)). Considering the size of the EM problem, it is obvious that introducing an equivalent circuit for modelling the cavities will be of substantial help in modifying the existing couplers and evaluating their performance. This approach will be examined in this paper while the implementation of the resulting equivalent circuit for the coupler modification will be shown elsewhere.

A literature survey shows that in most cases investigations of periodically loaded waveguides are accompanied by equivalent circuit modelling. Various examples of these efforts can be found in [2] where an extensive bibliography of the periodically loaded waveguides introduced before 1960 is presented. Also in [3]-[5], various structures for application in travelling wave tubes (TWTs) and linear accelerators are shown. In the TWT research community, the equivalent circuit modelling was widely employed as it provides the possibility of using Pierce approximate approach for

\footnotetext{
${ }^{1}$ European Organization for Nuclear Research

${ }^{2}$ The future configuration consists of two four-section and four three-section cavities, the changes to the current configuration is according to the requirements of LIU project.
}

calculating the gain of a structure. In the accelerator research community, however, the equivalent circuit modelling provides guidelines through which the adjacent dispersion curves of the periodic structure can be combined to realize the so-called confluence of the two passbands at the $\pi$-mode operation. However, the advent of $\pi / 2$-mode accelerating structures with substantially larger bandwidths reduces the need for such considerations. The equivalent circuit modelling was also implemented to model the higher order hybrid mode of an accelerator cavity in which single-cells are perturbed (via damping and detuning) to suppress the unwanted effects of the hybrid modes ([6]). In most publications, the equivalent circuit parameters are extracted by fitting to the dispersion curves instead of extracting the parameters from the different parts of the single-cell. In this paper, however, an approach for extracting the parameters of the equivalent circuit will be presented which is closely related to the physics of the problem and conventional data fitting approaches will not be employed. In section II, the accelerating structure of the SPS is introduced. The nature of the fields and the corresponding dispersion diagrams together with approaches for extracting the equivalent circuit parameters are presented. In the third section of the paper, the equivalent circuit of a periodic structure for a general case will be presented. Section IV shows the development of an equivalent circuit model for the accelerating structure. A brief conclusion will be presented in section $\mathrm{V}$.

\section{Parallel Bar Accelerating Structure of the SPS AND ITS $628 \mathrm{MHZ} \mathrm{HOM} \mathrm{COUPLER}$}

Figure 1 shows a single-cell of the $200 \mathrm{MHz}$ travelling wave cavity (also called the multi-stem structure). It consists of a central drift tube held by two stems (bars) that are connected to the circular outer enclosure through pedestals. The dimensions of the single-cell are as follows: $r_{a}=375 \mathrm{~mm}$, $r_{d}=130 \mathrm{~mm}, r_{s}=43.25 \mathrm{~mm}, r_{p}=221 \mathrm{~mm}, l_{u}=374 \mathrm{~mm}, l_{d}=150$ $\mathrm{mm} l_{s}=320 \mathrm{~mm}$. Figure 2 shows the dispersion curve of the fundamental mode and the unwanted HOM (the dispersion curves are extracted with the step of $\pi / 11$, the behaviour of the HOM dispersion curve at around $114^{\circ}$ phase advance will be examined later in this section.). In order to provide a larger bandwidth in comparison with the conventional $\pi$-mode, the operating mode is selected to be the $\pi / 2$-mode of the lowest frequency passband. The azimuthal profile of this mode at the 
centre of the unit-cell is shown in Fig. 3 (a). As can be concluded from the field profiles, the characteristics of the operating mode is also governed by the dimensions of the stems. This accelerating mode lies below the conventional $\mathrm{TM}_{010}$-mode (with the frequency of $307 \mathrm{MHz}$ for the unperturbed circular cavity) and therefore provides the size reduction possibility while supporting the strong axially directed field required for the acceleration. As can be seen from Fig. 2, $628 \mathrm{MHz}$ also corresponds to a $\pi / 2$ phase shift between the adjacent cells and as is seen in Fig. 3 (d), it has a quite strong axially directed electric field which shows its strong and unwanted interaction with the particle beam. In contrast to the dispersion curve of the fundamental mode which shows the typical behaviour of a narrowband periodic structure in which the group velocity does not change its sign, the HOM shows a region with different sign of group velocity. This confirms the presence of coupling between this mode and other HOMs. Careful examination of this coupling shows that the dominant $\mathrm{HOM}$ is of $\mathrm{TE}_{11}$ nature and that the coupling occurs mainly between the electric field of this mode and that of the unwanted HOM at the centre of the single-cell (see Fig. 3 (c) and (e)). As is shown in Fig. 3 (f), the TE 11 -type HOM has no harmful axially directed electric field which can perturb the particles, and therefore we will neglect the effect of the coupling with this mode as a first step towards the development of an equivalent circuit model. Another interesting property of the parallel bar structure is the fact that it is free of end effects. In this case by terminating a chain of $N$ unit-cell with copper plates at both ends, the resulted $N$ resonant frequencies correspond to the $n \pi / N(n=0,1, \ldots, N-1)$ phase shifts across an ideal unit-cell which is a part of an infinitely long periodic structure. As is seen in Fig. 2, the simulation results on a single section (consisting of 11 singlecells) which is terminated at both ends with metallic plates, confirm the absence of the end effects. A picture of a spare single section is shown in Fig. 4.

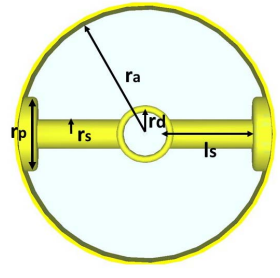

(a)

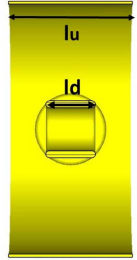

(b)
Fig. 1. Single-cell of the accelerating cavity: (a) front view, and (b) side view.

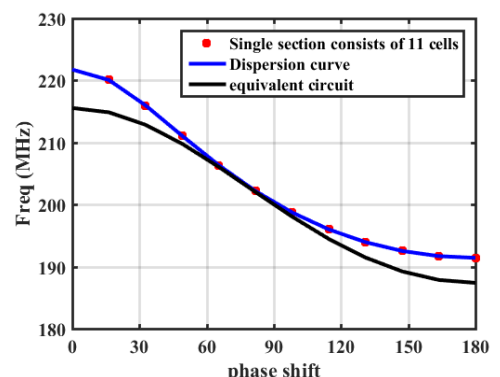

(a)

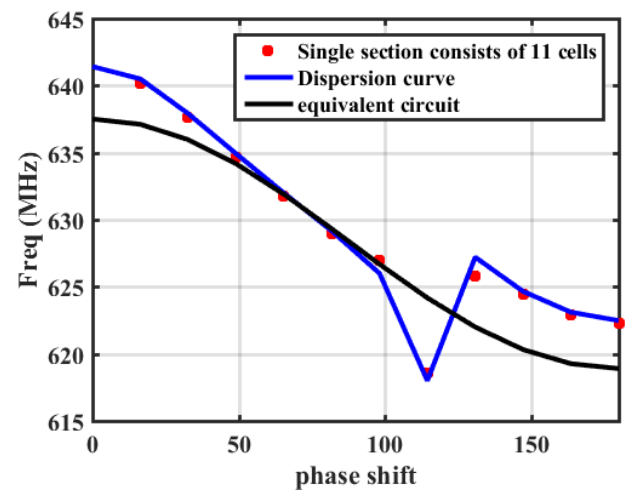

(b)

Fig. 2. Dispersion curves of the fundamental passband (a), and HOM (b).

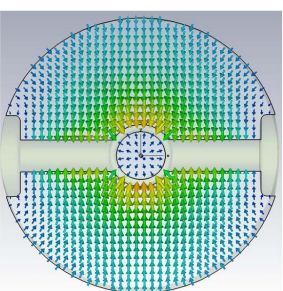

(a)

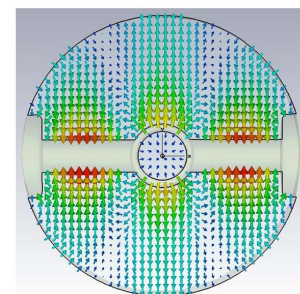

(c)

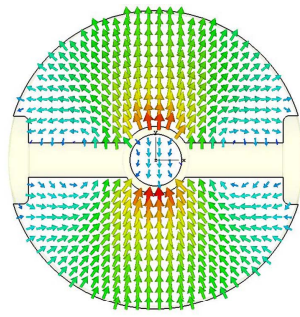

(e)

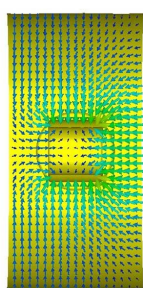

(b)

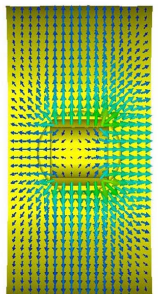

(d)

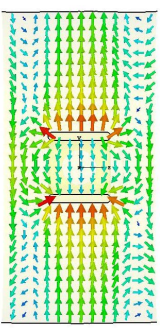

(f)

Fig. 3. Electric field patterns (using CST simulations) of (a) and (b) the operating mode $(f=200 \mathrm{MHz}),(\mathrm{c})$ and $(\mathrm{d})$ of the HOM $(f=628 \mathrm{MHz})$, and (e) and (f) of the TE 11 -type $\operatorname{HOM}(f=631 \mathrm{MHz})$.

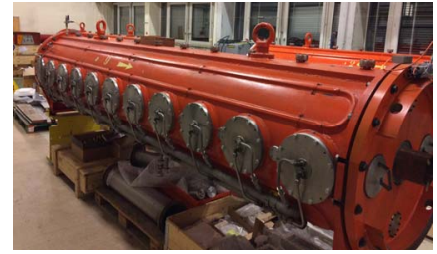

Fig. 4. A spare single section consisting of 11 single-cells and the inside-view of the section.

Fig. 5 (a) shows the HOM coupler inside one single-cell and (c) the arrangement of couplers inside one section. The 
HOM coupler itself consists of a $50 \Omega$ load and a frequency selective part which acts as a short circuit at $200 \mathrm{MHz}$ and as an open at $628 \mathrm{MHz}$ (see Fig. 7).

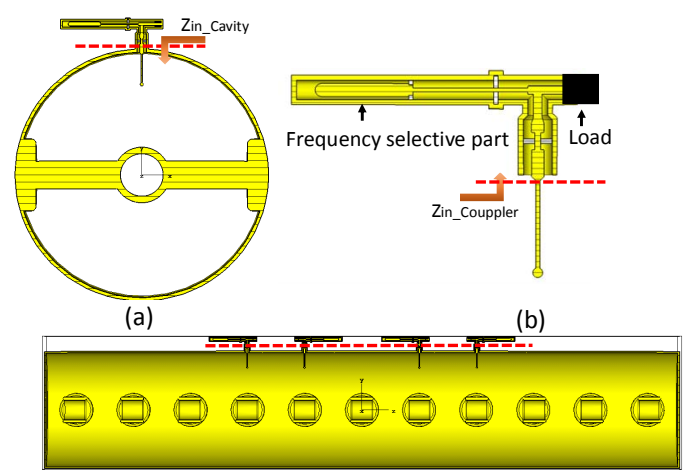

(c)

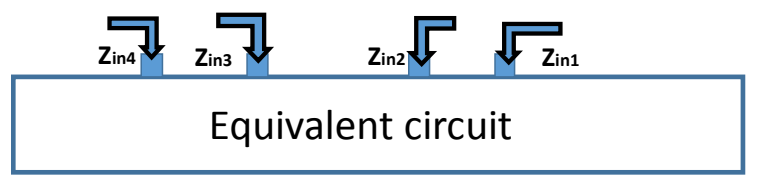

(d)

Fig. 5. (a) $628 \mathrm{MHz}$ HOM coupler inside one single-cell, (b) $628 \mathrm{MHz} \mathrm{HOM}$ coupler, (c) arrangement of HOMs for one section (the planes from which the input impedances will be calculated are shown in red), and (d) the equivalent circuit representing the section.

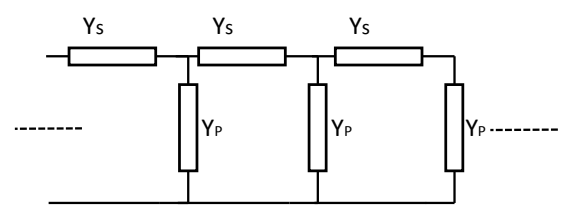

Fig. 6. General representation of a periodic structure.

\section{EQUivalent CIRCUIT MODELLING; GENERAL FORMULATION}

An arbitrary periodic structure can be represented by the equivalent circuit shown in Fig. 6. Considering a theta phase advance between two consecutive single-cells, it can be shown that [5]:

$$
\cos \theta=1+\frac{Y_{P}}{2 Y_{S}},
$$

where $Y_{P}$ and $Y_{S}$ are the series and the shunt admittances of the single-cell (see Fig. 6). Now according to the nature of the problem at hand, $Y_{P}$ and $Y_{S}$ are shaping a resonant circuit (of shunt or series type) corresponding to a specific mode and coupling between the single-cells across the periodic structure. This coupling can be of inductive, capacitive, or mixed type (the latter is also called a resonant coupling). In the next section, we will select a combination which can replicate the properties of the parallel bar accelerating periodic structure of the SPS.

Considering the fact that the input impedance of the HOM coupler (seen from the plane shown in Fig. 5 (b)) can be calculated analytically (Fig. 7), equivalent circuit representation of the section shown in Fig. 5 (d) provides the possibility of reducing the problem of optimizing the HOM coupler to a network synthesis problem with the goal of maximizing the absorbed power in the loads of the HOM coupler.

\section{EQuivalent CIRCUIT MODElling OF THE PARALlel BAR STRUCTURE}

An equivalent circuit of an EM problem depends not only on the nature of the fields but also on the way how a port interacts with the EM field. Considering 1) the planes at which the input impedances are to be calculated, (see Fig. 5 (c)), 2) the appearance of the electric field for both the fundamental and the HOM which can be represented by a capacitive effect (see Figs. 3 (a) and (c)), and 3) the surface current on the bars, it can be concluded that the equivalent circuit of the resonator is a shunt $L C$ circuit. This shunt circuit should be considered in the parallel branch of the equivalent circuit, i.e. $Y_{p}$ in Fig. 6 should be replaced with a shunt $L C$ circuit. Using an excitation scheme of Fig. 8 and examining the input impedance is the best way to confirm the accuracy of the above-mentioned assumptions. According to the equivalent circuit of this excitation scheme (the resonator model and the effect of probe which is modelled by a series capacitance) we expect the presence of two resonances at around $628 \mathrm{MHz}$, one of parallel nature which corresponds to the resonator and one of series nature which corresponds to the effect of the excitation probe and has a slightly lower frequency than the first resonance. This behaviour is observed in the simulated input impedance (see Fig. 9) and therefore assuming a parallel type resonator is in agreement with our observations. As already mentioned, the resonant frequency at $631 \mathrm{MHz}$ corresponds to the $\mathrm{TE}_{11}$-like HOM.

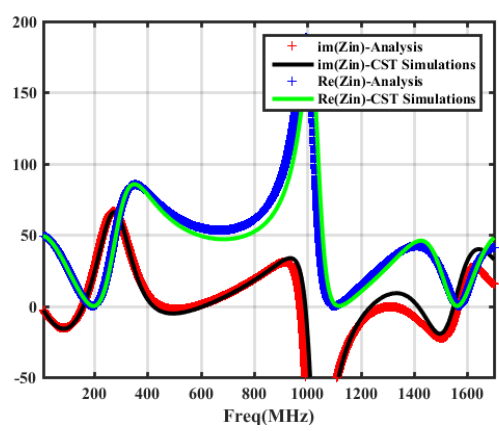

Fig. 7. Real and imaginary part of the input impedance of the coupler using an analytical approach and CST-simulations.
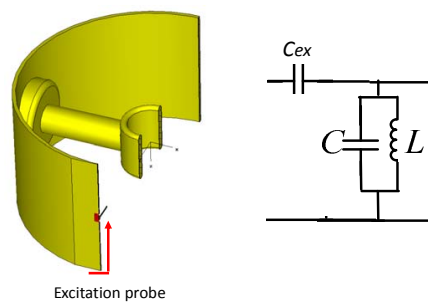

Fig. 8. Excitation scheme for a single-cell and its equivalent circuit. 


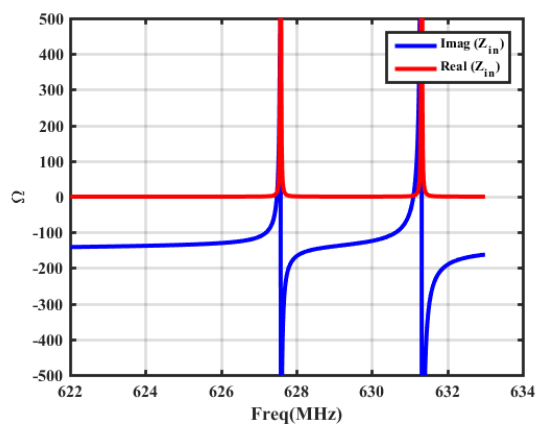

Fig. 9. Input impedance considering the excitation scheme shown in Fig. 8.

It can be shown that the characteristic impedance $\left(Y_{c}\right)$ of a shunt resonator at the resonant frequency can be calculated using the following equations:

$$
Y_{c}=\sqrt{\frac{L}{C}}=\left.\frac{\omega}{2} \frac{\partial Y_{\text {in }}}{\partial \omega}\right|_{\omega=\omega_{0}}, \omega_{0}=\frac{1}{\sqrt{L C}},
$$

where $Y_{\text {in }}$ is the input admittance of a resonator when it is weakly excited (for example as shown in Fig. 8). Now considering Eq. (2), both $L$ and $C$ of the equivalent circuit can be calculated. A shunt resistance is added to the resonators of Fig. 8 in order to consider the effect of limited quality factor. The next step is determining the coupling scheme between the unit-cells. Fig. 10 shows the equivalent circuit of a periodic arrangement of single-cells. In this representation, paralle resonators are connected through impedance inverters $(\lambda / 4$ transmission line with an impedance of $K$ ). For sufficiently narrowband structures, these inverters can be replaced by a capacitive $\pi$-network or an inductive $T$-network [7]. Figure 11 shows the equivalent circuit for narrow bandwidths. An impedance transformer with $n=-1$ (see Fig. 11), can be introduced to consider the 180 degree phase shift in the coupling element $\left(C_{m}\right)$. Using (1) and considering the equivalent circuit of Fig. 11, the dispersion relation can be calculated from the following equation:

$$
\frac{\omega_{0}^{2}}{\omega^{2}}=1-2 k n \cos \theta, \quad k=\frac{C_{m}}{C} .
$$

According to this equation, $\omega_{0}$ should be the radian frequency of the $\pi / 2$-mode which is consistent with our initial assumptions. Considering the fact that the $\pi$-mode resonant frequency is the smallest in the passband (see Fig. 2 (b)), $n$ should be 1 which is in accordance with the absence of a phase shift due to the coupling. The coupling factor can be calculated using the following equation

$$
k=\frac{f_{r}^{2}}{4}\left[\frac{1}{f_{\pi}^{2}}-\frac{1}{f_{0}^{2}}\right],
$$

where $f_{\pi}$ and $f_{0}$ are the frequencies of the $\pi$-mode and the 0 -mode, respectively. Comparison of the dispersion curve obtained from (4) and that of the actual model for both fundamental and HOM (see Fig. 2) shows that around the phase difference of about $\pi / 2$, the equivalent circuit has a quite good accuracy ( $k$ for the fundamental mode and the HOM is 0.014 and 0.06956 , respectively). Considering (2) and (4), and the quality factor of the HOM (35000), characteristics of the resonator can also be calculated ( $L=0.0264 \mathrm{nH}, C=2.43$ $\mathrm{nF}$, and $R=3.652 \mathrm{k} \Omega$ ). This step determines all the unknown parameters of the equivalent circuit.

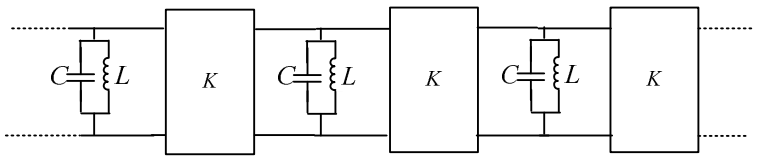

Fig. 10. Equivalent circuit of the coupled single-cells where the coupling element is an impedance inverter with an impedance of $\mathrm{K}$

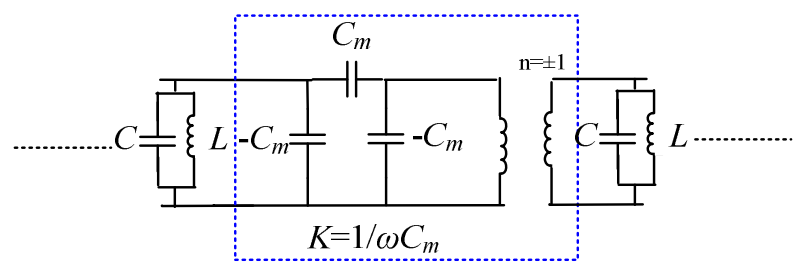

Fig. 11. Representation of impedance inverters for narrow bandwidths.

\section{CONCLUSIONS}

In this paper, an equivalent circuit model for the HOMs of the $200 \mathrm{MHz}$ parallel bar accelerating structure of the CERN-SPS is introduced. This model is able to replicate the dispersion diagram of the HOM. The parameters of the equivalent circuit model and their arrangement are determined according to the physics of the problem.

\section{ACKNOWLEDGMENT}

The authors are thankful to Dr. Erk Jensen from CERN for his many helpful comments. Patrick Kramer is also grateful for the support of the Federal Ministry of Education and Research of Germany.

\section{REFERENCES}

[1] E. Shaposhnikova et al., "Removing Known SPS Intensity Limitations for High Luminosity LHC Goals," in Proc. of IPAC'16, Busan, Korea.

[2] A. F. Harvey, "Periodic and Guiding Structures at Microwave Frequencies," in IRE Transactions on Microwave Theory and Techniques, vol. 8, no. 1, pp. 30-61, January 1960.

[3] M. A. Allen, G. S. Kino, "On the Theory of Strongly Coupled Cavity Chains," in IRE Transactions on Microwave Theory and Techniques, vol. 8, no. 3, pp. 362-372, May 1960.

[4] M. Chodorow and E. J. Nalos, "The Design of High-Power TravelingWave Tubes," in Proceedings of the IRE, vol. 44, no. 5, pp. 649-659, May 1956.

[5] T. M. Reeder, "An Equivalent Circuit for the "Centipede" Waveguide," in IEEE Trans. on Microwave Theory and Techniques, vol. 14, no. 4, pp. 200-205, Apr 1966.

[6] R. M. Jones et al., "Equivalent Circuit Analysis Of The SLAC Damped Detuned Structure," in Proc. of the Fifth European Particle Accelerator Conference, Sitges (Barcelona), vol. 2, pp. 1292-1294, 1996.

[7] J. Hesajn, Synthesis of Lumped Element, Distributed and Planar Filters, New York: Mc-Graw-Hill, 1990 